\title{
Model-Based FDI Applied to a Piezoelectric Active Vibration Suppression System for Smart Flexible Spacecraft
}

\author{
Paolo lannelli ${ }^{1}$ (D) Federica Angeletti ${ }^{1}$ (D) Paolo Gasbarri ${ }^{2}$ D
}

Received: 5 April 2021 / Revised: 27 April 2021 / Accepted: 3 May 2021 / Published online: 17 May 2021

(c) The Author(s) 2021

\begin{abstract}
Fault Detection and Isolation (FDI) techniques have captured extensive interest and attention in modern autonomous systems; in particular, they are of foremost importance in space applications, due to their scientific relevance, cost and current inability of doing on-orbit maintenance of space systems. In this scenario, FDI strategies are required to counteract possible failure events that, if not properly handled, can reduce system performance or compromise the realization of the mission objectives. In this paper, a model-based FDI strategy is implemented onboard a satellite equipped with a very large mesh reflector on which a distributed network of smart actuators/sensors is mounted to actively counteract undesired elastic vibrations. In particular, the detection and isolation of a possible piezo-actuator failure occurring in the Active Vibration Control (AVC) system of the antenna is addressed by a bank of Unknown Input Observers (UIOs). The design of the proposed UIOs is derived by solving a Linear Matrix Inequality (LMI) problem, which provides the conditions for their existence, and it is based on the linearized 3D state-space model of the controlled spacecraft, under the assumption that all the uncertainties, exogenous disturbances and measurement noises are neglected. Furthermore, pole assignment in the sense of D-stability is integrated in the standard formulation of the UIO to guarantee an adequate transient behaviour of the observers. Finally, an extensive Monte Carlo simulation campaign is conducted to assess the effectiveness of the proposed FDI architecture and its robustness against modelling uncertainties and measurement noise.
\end{abstract}

Keywords Large flexible space structures · Fault Detection and Isolation · Vibration control · Piezoelectric transducers · Unknown Input Observer · Linear Matrix Inequality

\section{Introduction}

Modern Earth Observation (EO) satellites are required to fulfil high demanding stability and pointing performance to benefit by the high-resolution capacity of their new instruments. In addition, large flexible appendages (as antennas and solar panels) are currently equipped to such spacecraft, thus demanding for the capacity of performing fast and precise manoeuvres without provoking excessive elastic displacement of such scientific instruments. These large systems-due to their scientific relevance, cost and extremely

Paolo Iannelli

paolo.iannelli@uniroma1.it

1 Department of Mechanical and Aerospace Engineering (DIMA), Sapienza University of Rome, Via Eudossiana 18, 00184 Roma, Italy

2 School of Aerospace Engineering, Sapienza University of Rome, Via Salaria 851, 00138 Rome, Italy limited possibilities of maintenance and repair-are good candidate to gain significant advantage from Fault Detection and Isolation (FDI) techniques.

A detailed knowledge of the failure process and an early detection of malfunctions or faults is a mandatory requirement for such safety critical systems a prompt awareness of failures appearance can effectively help in re-designing operations to avoid any loss of system performance or potential instability phenomena. In most applications, FDI functionalities are provided to the system via hardware redundancy [1]; however, although the use of these methods is common, the main drawbacks associated to them are the added complexity and costs resulting from the weight and volume of the redundant units. To overcome these critical disadvantages a wide variety of model-based FDI strategies, exploiting the mathematical model of the monitored system to make a fault/no-fault decision, has been extensively studied [2,3].

FDI methods have received considerable attention in the past two decades for several applications in the aeronautic 
and aerospace fields; in particular, many studies and FDI techniques have been developed for the Attitude and Orbital Control Subsystem (AOCS) of spacecrafts. In [4], a robust fault detector composed by a $\mathrm{H}_{-} / \mathrm{H}_{\infty}$ dynamical filter, associated to a GRL test for decision making, and a bank of Unknown Input Observers (UIOs) was proposed for thruster fault isolation in the context of Mars Sample Return Mission. The work reported in [5] addresses too the terminal rendezvous sequence of the Mars Sample Return mission and proposes a comparative study between position and attitude model-based FDI via Eigenstructure Assignment (EA) for thrusters' FDI. In [6] a bank of interacting multiple model unscented Kalman filters is, instead, developed to detect and isolate reaction wheel failures based on a highfidelity nonlinear model of the device.

Additionally, also the application of learning-based algorithms as a viable FDI solution has received particular attention. In this regard, one can mention: the Iterative Learning observer proposed in [7] to achieve estimation of time-varying thruster faults; the study of Patton et al. [8] where a robust approach to FDI for non-linear systems using neuro-fuzzy observers as a combination of the UIO and neuro-fuzzy structures, called the Optimal Robust Disturbance Decoupling Observer (ORDDO), is developed and [9], where a hierarchical methodology consisting of three combined Neural Network FDI schemes is used for pulsed plasma thrusters failure in formation flying satellites.

However, most of such studies have been performed with regard to the problem of satellite AOCS faults and little work has been conducted in FDI for active vibration control applications in space system. This is because the flexible dynamics of continuous systems, such as beams and plates or more complex structural systems, require high-order models to ensure an accurate dynamical representation and many FDI techniques become intractable for high-order system models [10]. The objective of this paper is to address the design of a model-based FDI architecture, based on a bank of UIOs, able to detect and isolate, with adequate responsiveness, a piezo-actuator failure occurring in the Active Vibration Control (AVC) system during classical attitude manoeuvres.

Section 2 presents a brief description of the basic structure and capabilities of the Unknown Input Observer (UIO), the mathematical formulation to synthetize this particular observer and its application in FDI problems. In Sect. 3, first, a Large Mesh Reflector Model (LMRM) is introduced as study case for the proposed FDI strategy. The considered Active Vibration Control (AVC) system is realized by a distributed network of five collocated and optimally placed piezoelectric sensors and actuators. Secondly the equations describing the tri-dimensional dynamics of a spacecraft equipped with flexible appendages are reported and their linearization around an operative point introduced. In Sect. 4, the structure of the bank of UIOs is specialized in the context of possible piezo-actuator failure occurring in the AVC system of the antenna. The effectiveness of the proposed FDI architecture and its robustness against modelling uncertainties and measurement noise is, then, assessed and discussed based on a Monte Carlo campaign by simulating a typical spacecraft attitude manoeuvre profile. Finally, concluding remarks on the presented method are discussed and future developments proposed in the last section.

\section{Model-Based FDI}

A model-based FDI architecture is generally composed of two-stages structure as shown in Fig. 1: (1) a residual generator (i.e. a detection filter structured in such a way to produce non-zero residual signals whenever a failure is occurring) and (2) a decision making process applied on the residual signals to discriminate the failure from false alarms caused by the presence of noise or other disturbances. This step could consist of simple threshold tests [11] or it may be a statistical decision method such as the Generalized Likelihood Ratio (GLR) test [12] or the Sequential Probability Ration Test (SPRT) [13]. Generally, the fault information extracted from this supervision module is then used to actively change the controller so that stability and acceptable performance of the entire system can be still verified.

In this section, we will firstly present the structure of the UIO and describe the conditions in terms of Linear Matrix Inequality (LMI) that provide the existence of the UIO for linear systems. Furthermore, the D-stability concept is integrated in the standard formulation of the UIO to adjust the dynamics of the observer. Afterwards, it is shown how multiple UIOs can be integrated together into a bank of observers to provide the system with FDI functionalities.

\subsection{Formulation of the Linear Unknown Input Observer (UIO)}

Consider the following general description of the continuous Linear Time Invariant (LTI) system:

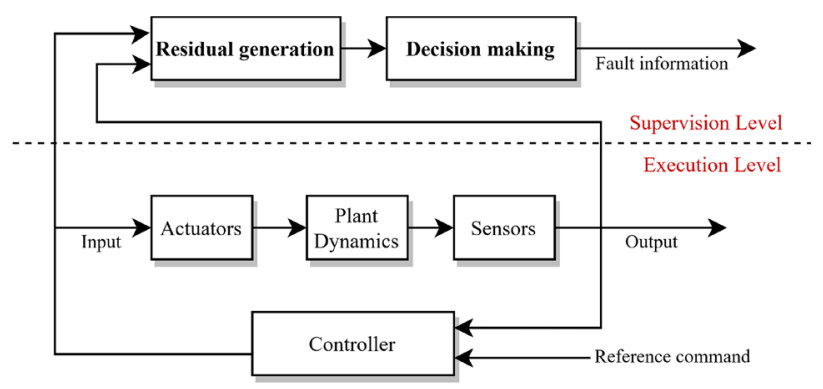

Fig. 1 General architecture of a model-based fault diagnosis system 


$$
\begin{array}{r}
\dot{x}(t)=\mathbf{A} x(t)+\mathbf{B} u(t)+\mathbf{D} v(t) \\
y(t)=\mathbf{C} x(t)
\end{array}
$$

where besides $x(t), u(t), y(t)$, that are the state vector, the known input vector, and the output vector of the plant respectively as in Eq. 1, an unknown input vector $v(t)$, which will be the unknown fault vector later on, is present in the dynamics of the system. Without loss of generality, it is assumed that $\mathbf{D}$ is a full column rank matrix.

An observer is defined as UIO for the system in Eq. 1 if its state estimation error vector approaches zero asymptotically, regardless of the presence of the unknown input vector in the system (e.g. a certain disturbance input from which the observer's output is desired to be independent). By assuming no a priori knowledge of the unknown inputs the UIO structure, in contrast to traditional Luenberger observers, is more suitable for use in fault detection applications. Although the actual unknown input itself does not need to be known, the primary requirement for a UIO or other disturbance decouplingbased observer is to have knowledge of the input distribution matrix $\mathbf{D}$.

Let us consider an observer, shown in Fig. 2, with the following structure:

$$
\begin{aligned}
& \dot{z}(t)=\mathbf{N} z(t)+\mathbf{H} u(t)+\mathbf{L}_{1} y(t) \\
& \hat{x}(t)=z(t)-\mathbf{L}_{2} y(t)
\end{aligned}
$$

where $\hat{x}$ is the state estimate vector, $z$ is the state vector of the UIO and the matrices $\mathbf{N}, \mathbf{H}, \mathbf{L}_{1}$ are defined as follows [14]:

$$
\begin{aligned}
\mathbf{N} & =\left(\mathbf{I}+\mathbf{L}_{2} \mathbf{C}\right) \mathbf{A}-\mathbf{K C} \\
\mathbf{H} & =\left(\mathbf{I}+\mathbf{L}_{2} \mathbf{C}\right) \mathbf{B} \\
\mathbf{L}_{1} & =\mathbf{K}\left(\mathbf{I}-\mathbf{C L}_{2}\right)-\left(\mathbf{I}+\mathbf{L}_{2} \mathbf{C}\right) \mathbf{A} \mathbf{L}_{2}
\end{aligned}
$$

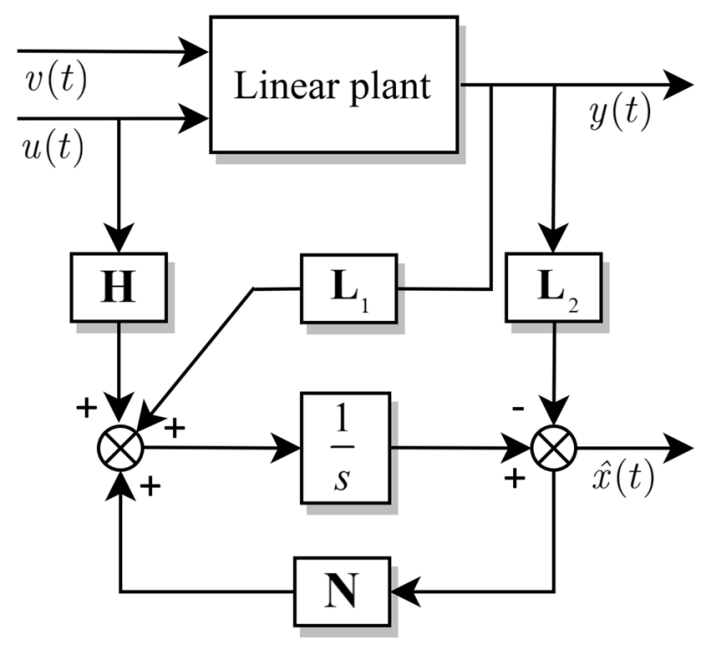

where I denotes the identity matrix with compatible dimensions with the expressions in Eq. 3 and the matrices $\mathbf{K}, \mathbf{L}_{2}$ which are design matrices used to guarantee the desired state estimation error dynamics of the observer. The following theorem, extracted from [14], is a sufficient condition of the existence of the UIO and gives us a systematic procedure to generate the observer based on the solution of a LMI problem in Eq. 4.

Theorem 1 Assume that $\mathbf{D}$ is of full column rank and that $\operatorname{rank}(\mathbf{C D})=\operatorname{rank}(\mathbf{D})$. An UIO, with state matrix $\mathbf{N}$ Hurwitz and with $e_{y}=\mathbf{C}(\hat{x}-x)$ tending to zero asymptotically for any initial value of $e_{y}(t=0)$, exists if and only if there exists a Lyapunov matrix $\mathbf{P}=\mathbf{P}^{T}>0$ (symmetric and definite positive), $\overline{\mathbf{Y}}$ and $\overline{\mathbf{K}}$ of adequate dimension so that:

$$
\begin{aligned}
((\mathbf{I}+\mathbf{U C}) \mathbf{A})^{T} \mathbf{P}+\mathbf{P}(\mathbf{I}+\mathbf{U C}) \mathbf{A}+(\mathbf{V C A})^{T} \overline{\mathbf{Y}}^{T} \\
+\overline{\mathbf{Y}}(\mathbf{V C A})-\mathbf{C}^{T} \overline{\mathbf{K}}^{T}-\overline{\mathbf{K}} \mathbf{C}<0
\end{aligned}
$$

with

$$
\begin{aligned}
\mathbf{Y} & =\mathbf{P}^{-1} \overline{\mathbf{Y}} \\
\mathbf{K} & =\mathbf{P}^{-1} \overline{\mathbf{K}} \\
\mathbf{U} & =-\mathbf{D}(\mathbf{C D})^{+} \\
\mathbf{V} & =\mathbf{I}-(\mathbf{C D})(\mathbf{C D})^{+} \\
\mathbf{L}_{2} & =\mathbf{U}+\mathbf{Y} \mathbf{V}
\end{aligned}
$$

where the $(\mathbf{C D})^{+}$denotes the generalized pseudoinverse operator applied to the matrix $\mathbf{C D}$ given by $(\mathbf{C D})^{+}=\left((\mathbf{C D})^{T}(\mathbf{C D})\right)^{-1}(\mathbf{C D})^{T}$.

The previous formulation of the UIO was derived as the special (linear) case of the work done by Chen and Saif [14] for which a full-order nonlinear observer was constructed for a class of Lipschitz nonlinear systems with unknown inputs. However, direct application of the LMI condition may result in unsatisfactory dynamical behaviour of the state estimation error as stated in [15]. To overcome this problem the D-stability concept proposed in [16] can be used jointly with the previous theorem to constrain the poles of UIO (poles of matrix $\mathbf{N}$ ), as reported in Theorem 2.

Theorem 2 The eigenvalues of the observer state space matrix $\mathbf{N}$ can be assigned into a prescribed region $D=\bigcap_{k=1}^{n_{s}} D_{k}$ if there exists a common Lyapunov matrix $\mathbf{P}=\mathbf{P}^{T}>0$ and matrices $\overline{\mathbf{Y}}$ and $\overline{\mathbf{K}}$ such that the set of $n_{s}$ LMIs reported in Eq. 6 is simultaneously satisfied.

$$
\begin{aligned}
\boldsymbol{\alpha}_{k} & \otimes \mathbf{P}+\boldsymbol{\beta}_{k} \otimes\left(\mathbf{A}^{T} \mathbf{P}+(\mathbf{U C A})^{T} \mathbf{P}+(\mathbf{V C A})^{T} \overline{\mathbf{Y}}^{T}-\mathbf{C}^{T} \overline{\mathbf{K}}^{T}\right)+ \\
& +\boldsymbol{\beta}_{k}^{T} \otimes(\mathbf{P A}+\mathbf{P}(\mathbf{U C A})+\overline{\mathbf{Y}}(\mathbf{V C A})-\overline{\mathbf{K}} \mathbf{C})<0 k=1,2, \ldots, n_{s}
\end{aligned}
$$

Fig. 2 Schematics of an UIO applied to a LTI system 
where $\boldsymbol{\alpha}_{k}$ and $\boldsymbol{\beta}_{k}$ are matrices of appropriate dimension defining each region $D_{k}$. The symbol $\otimes$ represents the Kronecker product operator.

In the following study, the assigned LMI region for each UIO has been selected as the intersection of two elementary LMI region defined as follows:

- $D_{1}$ : disk centred at $\left(c_{d}, 0\right)$ and radius $r_{d}$.

- $D_{2}$ : left half plane delimited by the vertical line $\operatorname{Re}(z)<-\alpha$ with $\alpha>0$.

These parameters $\left(c_{d}, r_{d}, \alpha\right)$, that define the LMI region assignment approach, must be adjusted in such a way that the dynamics of the estimation error react quickly enough to any type of failure considered, allowing a first distinction between healthy/faulty devices.

\subsection{Fault Detection and Isolation: Bank of UIOs}

Similarly to [17], by exploiting the capability of the UIO to generate a state estimation error decoupled from one or more unknown input signals, it is possible to design a bank of observers so that the output of each of them is sensitive to every possible failure except one. Therefore, the UIO with the minimum estimation error signal in the sense of the L2-norm reveals that a fault has occurred in the associated actuator. Considering $s$ possible failure happening in the system, if for each one of them in possible to verify the rank condition in Theorem 1 then a bank of $s$ UIOs can be assembled as in Fig. 3.

The bank of UIOs takes as input the measured output of the system $y(t)$ and the output of the controller $u(t)$ (i.e. the undamaged input of the system) and produces as output

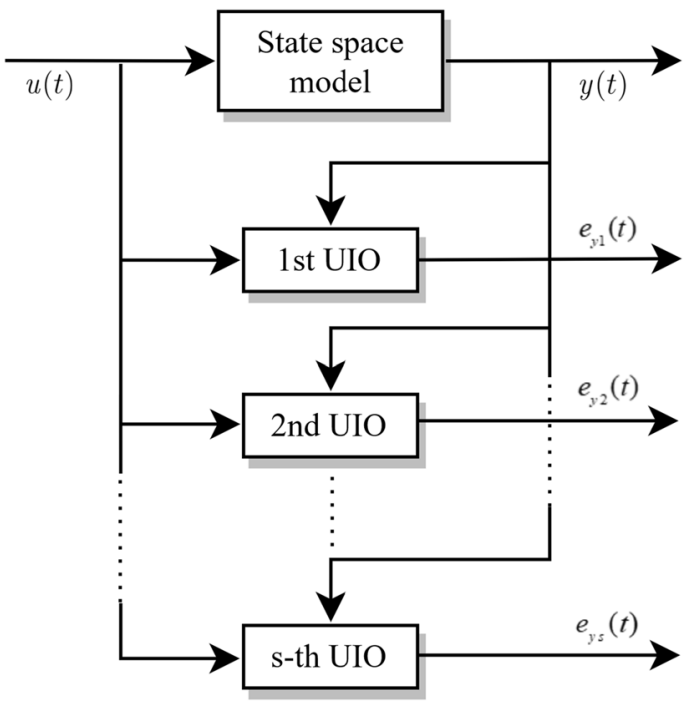

Fig. 3 Schematics of the bank of $s$ UIOs the estimation errors $e_{y i}(t)$ with $i=1,2, \ldots, s$. As described in Sect. 1, the output of the residual generator is used for the decision-making process to determine the presence and location of the faulty component. In this work, this process starts by computing the L2-norms of $e_{y i}(t)$.

In particular, both detection and isolation were tested using two similar procedures, one involving the L2-norm of the signals $\left\|e_{y i}(t)\right\|, i=1,2, \ldots, s$ and one involving the derivative of the L2-norm of the same signals $\left\|\dot{e}_{y i}(t)\right\|$, $i=1,2, \ldots, s$. In the detection phase, one of the following checks is implemented to verify the presence of an actuator failure:

o Procedure 1: each UIO is associated to a binary label $H_{N i}, i=1,2, \ldots, s$, which can be 1 if the related $\left\|e_{y i}\right\|$ is over a predefined threshold $T_{n}$ for an assigned time interval (defined as confirmation time $t_{c}$ ) or 0 in the other cases, as described in Eq. 7.

$$
H_{N i}=\left\{\begin{array}{ll}
1 & \text { if }\left\|e_{y i}(t)\right\|>T_{n} \text { for } t>t_{c} \\
0 & \text { else }
\end{array} i=1,2, \ldots, s .\right.
$$

If at least two out of the $s$ labels are equal to one, then a failure is detected by the system.

o Procedure 2: similarly to the previous procedure, if at least 2 out of all the $\left\|\dot{e}_{y i}\right\|$ are over a predefined threshold $T_{n}$ for the confirmation time $t_{c}$, then a failure is detected, as described in Eq. 8.

$$
H_{D i}=\left\{\begin{array}{ll}
1 & \text { if }\left\|\dot{e}_{y i}(t)\right\|>T_{n} \text { for } t>t_{c} \\
0 & \text { else }
\end{array} i=1,2, \ldots, s .\right.
$$

The threshold and the confirmation time are parameters that must be selected and tuned to balance the trade-off between fast confirmation of the failure and robustness to false alarms. Their selected values usually differ between Procedure 1 and Procedure 2.

In addition, to ensure the FDI architecture has also isolation capabilities, two alternative approaches can be used:

- Approach 1: Search for the minimum between all $\left\|e_{y i}(t)\right\|$ (or all $\left.\left\|\dot{e}_{y i}(t)\right\|\right)$. However, it should be noticed that such a method can lead to a higher rate of failed detection and false alarms in presence of noise (which cause the L2-norm of the signal to randomly change at different time instants);

- Approach 2: Verify which one of the $H_{N i}\left(\right.$ or $H_{D i}$ ) is not over the preselected threshold. In this case, if just one out of the $s$ labels is under the threshold (ideally equal to zero), the failed actuators is the one related to such UIO. This method can guarantee a higher rate of isolation in case of noise and uncertainties. 


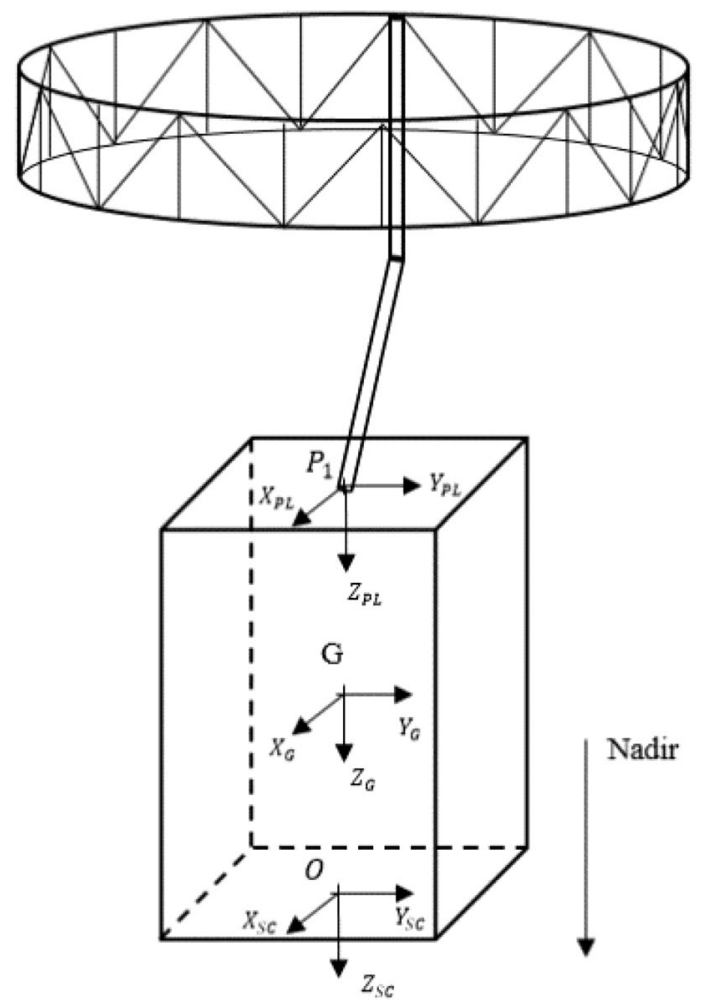

Fig. 4 Schematics of the spacecraft equipped with a large flexible mesh reflector

\section{Test Case: Spacecraft Equipped with a Large Mesh Reflector}

To test the proposed FDI architecture, the case of a spacecraft equipped with a large mesh reflector model (LMRM) is considered. In detail, the appendage is attached to a central platform-considered to be rigid with respect to the flexible structure-in correspondence of an attachment point $P_{1}$ defined in the $\mathrm{S} / \mathrm{C}$ reference frame, whose origin $O$ is located at the centre of the launch vehicle payload adapter (see Fig. 4). The inertial properties of the platform, without considering the appendage, are listed in Table 1.

\subsection{Large Mesh Reflector Structural Model}

The flexible appendage has been realized so to be representative (i.e. Model Replica) of the payload of a realistic space mission. Indeed, a structural model based on available information about the AstroMesh $®$ antenna [18, 19] has been realized in a finite element commercial environment [20]. To properly benefit from a distributed active vibration control system, the properties of the developed model have been modified to achieve a mass reduction of up to $30 \%$ with respect to the original AstroMesh® reflector. The main properties of the test case structure compared with AstroMesh® data and its replica the are reported in Table 2 .

To allow the in-orbit deployment of the reflector and to support the antenna during operations, an extendable boom is generally implemented on those spacecrafts equipped with such a type of scientific instrument. Therefore, an 8-m long, $20 \mathrm{~kg}$ boom has been included in the structural model of the LMRM, which has been chosen as test case. The first frequencies and modal shapes of the structure-compared with AstroMesh $®$ 's ones-can be found in Table 3.

Concerning the available data, the order of the modes for the AstroMesh ${ }^{\circledR}$ clamped system is: yaw, pitch and roll, while the related frequencies are not available. Nevertheless, having compared the behaviour of the mesh reflector with the original AstroMesh ${ }^{\circledR}$ model in Table 3, it is reasonable to assume their dynamic behaviour is quite similar.
Table 1 Platform inertial properties

\begin{tabular}{|c|c|c|c|c|c|c|c|c|c|}
\hline \multirow[t]{2}{*}{ Mass (kg) } & \multicolumn{6}{|c|}{ Inertia $\left(\mathrm{kg} / \mathrm{m}^{2}\right)$} & \multicolumn{3}{|c|}{$\mathrm{CoG}(\mathrm{m})$} \\
\hline & $\mathbf{J}_{x x}$ & $\mathrm{~J}_{y y}$ & $\mathrm{~J}_{z z}$ & $\mathrm{~J}_{x y}$ & $\mathrm{~J}_{x z}$ & $\mathrm{~J}_{y z}$ & $X$ & $Y$ & $Z$ \\
\hline 940 & 590 & 620 & 550 & 2 & 4 & -8 & 0 & 0 & -1 \\
\hline
\end{tabular}

Table 2 Reflector properties: comparison among different models

\begin{tabular}{llll}
\hline & AstroMesh® data & Model Replica & LMRM \\
\hline Mass $(\mathrm{kg})$ & Truss: 53 & Truss: 53.01 & Truss: 38.34 \\
& Mesh: 4.1 & Mesh: 4.25 & Mesh: 4.25 \\
& Total: 57.1 & Total: 57.26 & Total: 42.6 \\
1st Mode $(\mathrm{Hz})$ & Truss: 0.30 & Truss: 0.30 & Truss: 0.20 \\
& Truss + Mesh: 0.80 & Truss + Mesh: 0.78 & Truss + Mesh: 0.53 \\
\hline
\end{tabular}


Table 3 Comparison of clamped modes and frequencies

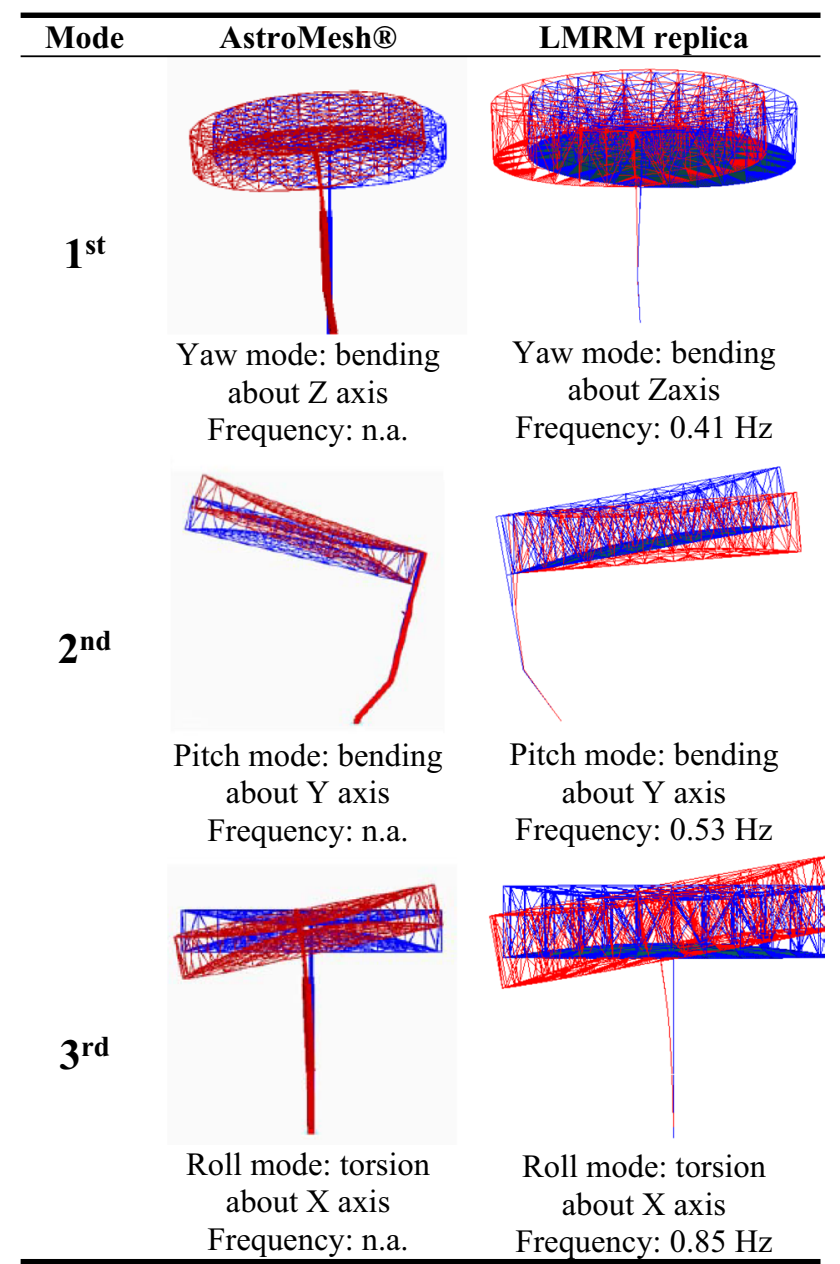

\subsection{Dynamics of the Assembled Spacecraft}

To simplify the design of both controller and observers, the nonlinear dynamic model has been linearized starting from the non-linear equations of motion for spacecraft attitude control problems. For brevity's sake, only the final equations are reported in this paper, as the steps leading to such formulation have already been derived in authors' previous works $[21,22]$. In general, a state vector $X=\left[X_{G}, \Theta, \eta\right]$ can be considered as including the position of the platform center of gravity $\mathrm{G}$ with respect to an ECI inertial frame $X_{G}$, the attitude of the body reference frame with respect to the inertial system $\Theta$ and the modal amplitudes of the equipped flexible appendage $\eta$. Hence, the non-linear dynamics of the system can be written as indicated in Eq. 9.

$\mathbf{M}_{T}^{G} \ddot{X}+\mathbf{C}_{T} \dot{X}+\mathbf{K}_{T} X+N_{L, T}=F_{T}^{G}$.

In detail, the system total mass matrix is reported in Eq. 10 as
$\mathbf{M}_{T}^{G}=\left[\begin{array}{ccc}\left(m_{A_{i}}+m_{b}\right) \mathbf{I} & { }^{B} \tilde{\mathbf{p}}_{G}^{A_{i} \times} & { }^{B} \mathbf{L}_{k}^{A_{i}} \\ \left({ }^{B} \tilde{\mathbf{p}}_{G}^{A_{i} \times}\right)^{T} & { }^{B} \tilde{\mathbf{J}}_{G}^{A_{i}} & { }^{B} \tilde{\mathbf{S}}_{k}^{A_{i}} \\ { }^{B} \mathbf{L}_{k}^{A_{i} T} & { }^{B} \tilde{\mathbf{S}}_{k}^{A_{i} T} & \mathbf{I}\end{array}\right]$

where $m_{b}$ is the mass of the platform, $m_{A_{i}}$ the mass of the appendage, ${ }^{B} \tilde{\mathbf{p}}_{G}^{A_{i} \times}$ is the skew matrix of the static moment vector of the system with respect to the platform center of gravity G, defined in the body reference frame, ${ }^{B} \tilde{\mathbf{J}}_{G, T O T}^{A_{i}}$ the moment of inertia of the system with respect to $G$ in the body frame, ${ }^{B} \mathbf{L}_{k}^{A_{i}}$ and ${ }^{B} \tilde{\mathbf{S}}_{k}^{A_{i}}$ translation and rotation modal participation factors (coupling with the rigid motion), $\mathbf{I}$ is the identity matrix. In addition, the matrices $\mathbf{C}_{T}$ and $\mathbf{K}_{T}$ are defined as

$\mathbf{C}_{T}=\left[\begin{array}{lll}\mathbf{0} & 0 & 0 \\ 0 & 0 & 0 \\ 0 & 0 & 2 \Sigma \Omega\end{array}\right], \mathbf{K}_{T}=\left[\begin{array}{ccc}0 & 0 & 0 \\ 0 & 0 & 0 \\ 0 & 0 & \Omega^{2}\end{array}\right]$,

where $\boldsymbol{\Omega}$ is a diagonal matrix listing all angular frequencies of the appendages as cantilevered to satellite and $\boldsymbol{\Sigma}$ is a diagonal matrix containing the $k$-th damping factor $\xi_{k}$ of the corresponding elastic mode. The term $F_{T}^{G}$ indicates the generalized forces (forces $f_{G}$, torques $c_{G}$ and projection of forces on the modal base $\tilde{f}^{e}$ ) applied to the spacecraft in G.

In this work, a network of smart actuators is implemented on the flexible structure. The forces exerted by the active devices on the modal base are included in the following expression:

$F_{T}^{G}=\left[f_{G}^{T}, c_{G}^{T},\left(\tilde{f}^{e}+\tilde{\mathbf{K}}_{U \Psi}^{g} \psi_{p}\right)^{T}\right]^{T}$,

where $\tilde{\mathbf{K}}_{U \Psi}^{g}$ is the electro-mechanical coupling matrix of the piezoelectric actuators projected on the modal basis and $\psi_{p}$ the input voltage vector [23]. The non-linear terms of Eq. 9 are reported below:

${ }^{B} N_{L}^{G}=\left[\begin{array}{l}\omega \wedge\left(\omega \wedge{ }^{B} \tilde{p}_{G}^{A_{i}}\right)+2 \omega \wedge \sum_{k=1}^{N}{ }^{B} L_{k}^{A_{i}} \dot{\eta}_{k} \\ \omega \wedge{ }^{B} \tilde{\mathbf{J}}_{G, T O T}^{A_{i}} \omega+\omega \wedge \sum_{k=1}^{N}{ }^{B} \tilde{S}_{k}^{A_{i}} \dot{\eta}_{k}+\sum_{k=1}^{N}{ }^{B} \tilde{\mathbf{J}}_{1}^{A_{i}, k} \dot{\eta}_{k} \omega \\ -\frac{1}{2} \omega^{T B} \tilde{\mathbf{J}}_{1}^{A_{i}, k} \omega\end{array}\right]$,

where $\omega$ is the angular velocity of the system with respect of the inertial system written in the body reference frame, ${ }^{B} \tilde{\mathbf{J}}_{1}^{A_{i}, k}$ is the variation of the inertia tensor due to the flexibility [22].

\subsection{Distributed Active Vibration Control (AVC) System}

In the proposed study, the LMRM is equipped with a distributed AVC system composed of five actuators and 
Table 4 Properties of the selected piezoelectric actuators and sensors

\begin{tabular}{lll}
\hline & Actuator & Sensor \\
\hline Young modulus $(\mathrm{GPa})$ & 100 & 100 \\
Dimensions $(\mathrm{m})$ & 0.1 (length) 0.013 (radius) & 0.1 (length) 0.01 (width) \\
Electromechanical coupling coefficient $d_{33}(\mathrm{~V} / \mathrm{m})$ & $400 e-12$ & - \\
Electromechanical coupling coefficient $d_{31}(\mathrm{~V} / \mathrm{m})$ & - & $180 e-12$ \\
Density $\left(\mathrm{kg} / \mathrm{m}^{3}\right)$ & 7000 & 7000 \\
Max $/ \mathrm{min}$ Actuation voltage $(\mathrm{V})$ & \pm 100 & -
\end{tabular}

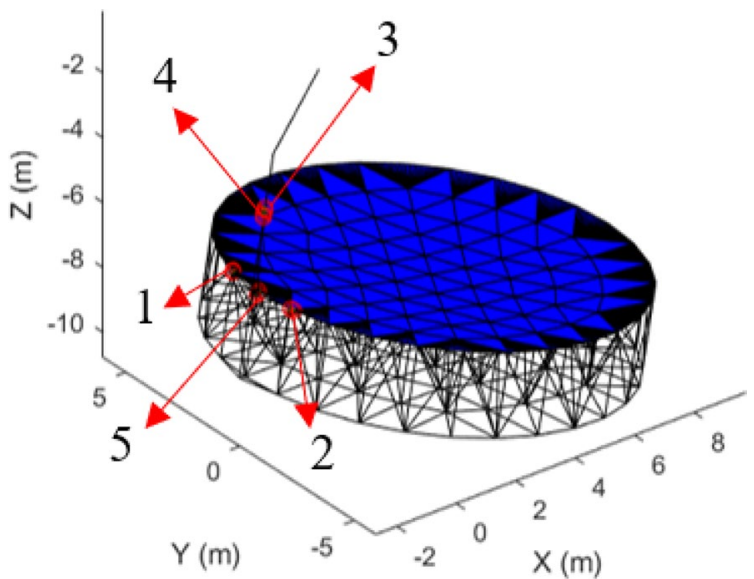

Fig. 5 Final configuration of co-located actuators/sensors [20]

sensors, whose properties are listed in Table 4. Their locations have been selected based on a placement procedure that made use of the controllability and observability gramians of the system to attempt to increase-from a control perspective - the relative controllability and observability of the most important system modes. More details about the placement process may be found in Authors' previous work [20]. The final configuration-position and related number of actuators and sensors-is reported in Fig. 5.

In particular a (pi)-shaped piezoelectric stack actuator (P-PSA) [24] was proposed to exert the strong actuating power of piezoelectric stack; when a driving voltage is applied to a piezoelectric stack, expansion or contraction along the axial direction of the stack will be induced because of the inverse piezoelectric effect. If the stack is constrained at its two ends to a host support, actuating moments will be generated at the constrained edges (see Fig. 6).

As reported in literature [24, 25], the bending moment generated by the actuator about the local Z-axis in Fig. 6 can be described as

$M=\frac{n_{l} d_{33} A_{P Z} E_{P Z}}{l_{P Z}} h_{a} \psi_{p}=c_{P Z} h_{a} \psi_{p}$,

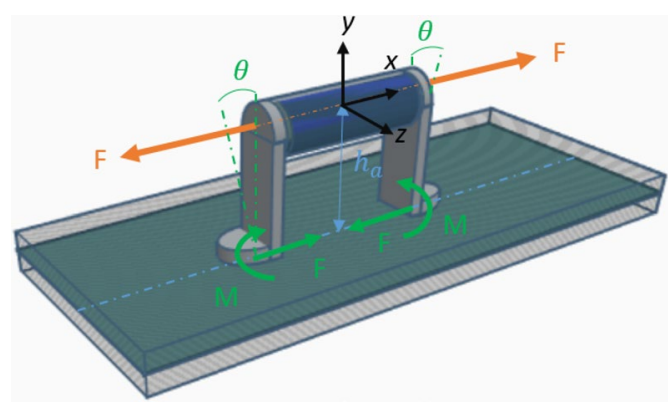

Fig. 6 P-PSA actuator/sensor setup [21]

where $h_{a}$ is the distance between the longitudinal axis of the actuator and the neutral plane of the passive structure, $\psi_{p}$ is the voltage needed to actuate the piezo device, $d_{33}$ is the piezoelectric material coefficient, $A_{P Z}, E_{P Z}, l_{P Z}$ and $n_{l}$ are the area, the Young module the length and the layer of the device respectively.

If we consider a beam element with two nodes $m$ and $n$ (three translations and three rotations each), the degrees of freedom to be considered will be

$V_{m n}=\left[u_{m}, v_{m}, z_{m}, \alpha_{m}, \beta_{m}, \theta_{m}, u_{n}, v_{n}, z_{n}, \alpha_{n}, \beta_{n}, \theta_{n}\right]$,

where $u, v, z$ indicate the translational degrees of freedom along the local $\mathrm{X}, \mathrm{Y}$ and $\mathrm{Z}$-axis in Fig. 6 respectively, while $\alpha, \beta, \theta$ the rotational ones. Consequently, the electromechanical coupling matrix for one smart actuator can be written in the element local 3-D reference frame as:

$\boldsymbol{K}_{U \Psi}^{e}=c_{P Z}\left[1,0,0,0,0,-h_{a},-1,0,0,0,0, h_{a}\right]^{T}$.

\subsection{Linearized Equations of Motion (LEoM)}

In this study, the orbital dynamics are assumed to have a negligible effect when the orbital period is very low with respect to the attitude controller bandwidth [26, 27] (i.e. when the duration of the manoeuvre is short with respect to the orbital period). Indeed, such a situation occurs in a major part of space applications, where the angular rates are not 
high enough to retain the gyroscopic stiffness term. In such conditions, the velocity, and eventually the operating point, can be assumed equal to zero. Therefore, by operating a Taylor expansion around the operating point, one can obtain

$$
\begin{aligned}
& \overline{\mathbf{M}}_{T} \ddot{X}+\overline{\mathbf{C}}_{T} \dot{X}+\overline{\mathbf{K}}_{T} X=F_{T}^{G} \\
& \dot{\theta}=\mathbf{Q}^{\theta}(\theta) \omega
\end{aligned}
$$

where the overline sign refers to the system written in linearized form and $\mathbf{Q}^{\theta}(\theta)$ is the matrix function of the rotation angles depending on the type of assumed rotation sequence. It can be demonstrated [28] that the mass and stiffness matrices correspond to the ones reported in Eq. 9, while the damping matrix, also multiplying the rate terms, may be expressed as depending on the Jacobian matrix of the gyroscopic term $\mathbf{H}_{j a c}$

$\overline{\mathbf{C}}_{T}=\left[\begin{array}{ccc}\mathbf{0} & \mathbf{0} & \mathbf{0} \\ \mathbf{0} & \mathbf{H}_{j a c} & \mathbf{0} \\ \mathbf{0} & \mathbf{0} & 2 \Sigma \boldsymbol{\Omega}\end{array}\right]$.

Therefore, according to the previous considerations, it is reasonable to assume

$$
\begin{aligned}
\mathbf{H}_{j a c} & =\left(\boldsymbol{\omega}^{\times}\right)_{\mid \omega=0}^{B} \mathbf{J}_{b}^{G}-\left({ }^{B} \mathbf{J}_{b}^{G} \omega\right)_{\mid \omega=0}^{\times}=\mathbf{0} \\
\dot{\theta} & =\left.\frac{\partial \mathbf{Q}^{\theta}(\theta)}{\partial \theta}\right|_{\substack{\theta=0 \\
\omega=0}} \omega_{o} \theta+\mathbf{Q}^{\theta}\left(\theta_{0}\right)\left(\omega-\omega_{o}\right)=\mathbf{I} \omega
\end{aligned}
$$

Finally, the linearized equations can be presented in the state-space standard form as:

$$
\begin{aligned}
X(t) & =\mathbf{A} X(t)+\mathbf{B} u(t) \\
Y(t) & =\mathbf{C} X(t)
\end{aligned}
$$

with:

$$
\begin{aligned}
& \mathbf{A}=\left[\begin{array}{cc}
\mathbf{0} & \mathbf{I} \\
-\overline{\mathbf{M}}_{T}^{-1} \overline{\mathbf{K}}_{T} & -\overline{\mathbf{M}}_{T}^{-1} \overline{\mathbf{C}}_{T}
\end{array}\right] \\
& \mathbf{B}=\left[\begin{array}{ll}
\mathbf{0} & \mathbf{B}_{v}^{T}
\end{array}\right]^{T} \quad \mathbf{C}=\left[\begin{array}{ll}
\mathbf{C}_{o x} & \mathbf{C}_{o v}
\end{array}\right] \\
& \mathbf{B}_{v}=\left[\begin{array}{ccc}
\mathbf{I} & \mathbf{0} & \mathbf{0} \\
\mathbf{0} & \mathbf{I} & \mathbf{0} \\
\mathbf{0} & \mathbf{0} & \mathbf{B}_{m}
\end{array}\right]
\end{aligned}
$$

Assuming the flexible structure is not subjected to forces other than the actuators actions, the input forces $u$ will include $u=\left[f_{g}^{T}, c_{g}^{T}, \psi_{p}^{T}\right]^{T}$ and the matrix $\mathbf{B}_{m}=\tilde{\mathbf{K}}_{v \psi}^{g}$. Given that a colocated active control system is considered in this study, and the output matrix $\mathbf{C}$ is $\mathbf{C}=\left[\begin{array}{ll}\mathbf{0} & \mathbf{C}_{o v}\end{array}\right]$. A voltage model amplifier is used to amplify the current signal from the piezoelectric sensor to measurable output voltage signal $\psi_{p s}$. As reported in [29], in this work, a high pass filter with a lower cut-off frequency of $0.1 \mathrm{~Hz}$ is used to model the voltage model amplifier circuit while the cut-off at high frequency has been neglected since it has no influence in this particular sensing application.

Figure 7 shows the complete closed-loop configuration of the spacecraft and the failure management unit made of a bank of five UIOs, each one of them prescribed to the isolation of the failure of one piezoelectric actuator.

To test the proposed FDI architecture, the spacecraft equipped with the flexible LMRM is controlled using a classical PID (Proportional-Integral-Derivative) attitude control system in parallel to a DVF (Direct velocity Feedback) vibration suppression system [30]. Delays equal to $0.1 \mathrm{~s}$ and $0.05 \mathrm{~s}$ are introduced respectively for the attitude command and piezo command with the transfer function matrices $\boldsymbol{G}_{\tau a}(s)$ and $\boldsymbol{G}_{\tau p}(s)$ using a 1/5 degrees Padé approximation [31].

Since the design of the UIOs is based on the state-space model of the assembled system under the assumption that all the uncertainties (as inertial properties, natural frequencies, damping and chain delays), exogenous disturbances (as disturbance forces and torques applied on the spacecraft) and measurements noise are neglected, in Sect. 4.4 a Monte Carlo simulations campaign has been carried out to evaluate the off-nominal performance of the proposed FDI approach.

To tackle the abovementioned uncertainties in an efficient manner, the uncertain state space model is put into the linear fractional representation (LFT) framework [32] as expressed in Eq. 22 and the uncertainty values are reported in Table 5.

$Y(t)=F_{u}(\boldsymbol{P}, \boldsymbol{\Delta})\left[\begin{array}{l}d \\ c_{g} \\ \psi_{p}\end{array}\right]+\left[\begin{array}{l}n_{\theta} \\ n_{\omega} \\ n_{\psi}\end{array}\right]$,

where $\left[n_{\theta}, n_{\omega}, n_{\psi}\right]^{T}$ is the output measurement noise vector and $F_{u}(\boldsymbol{P}, \boldsymbol{\Delta})$ is the upper LFT of system matrix $\boldsymbol{P}(s)$ with $\boldsymbol{\Delta}$ block-diagonal matrix containing the uncertainties extracted from the uncertain state space model of Eq. 20.

Besides the plant's uncertainties, also some random white noise is introduced in the measurements, with values reported in Table 6, to obtain more realistic output data.

\section{Simulation and Results}

In this study, the algorithms used to design the FDI system and to determine its overall performances are implemented in MATLAB/SIMULINK; in detail, the parameters of the bank are initialized in the YALMIP environment [33], a MATLAB toolbox for modelling and optimization extremely suited for applications in system and control theory. The parameters of each UIO, defined in Theorem 1 of Sect. 2.1 are computed using the semidefinite programming solver SDPT3 [34] called as external optimization routine by YALMIP. 


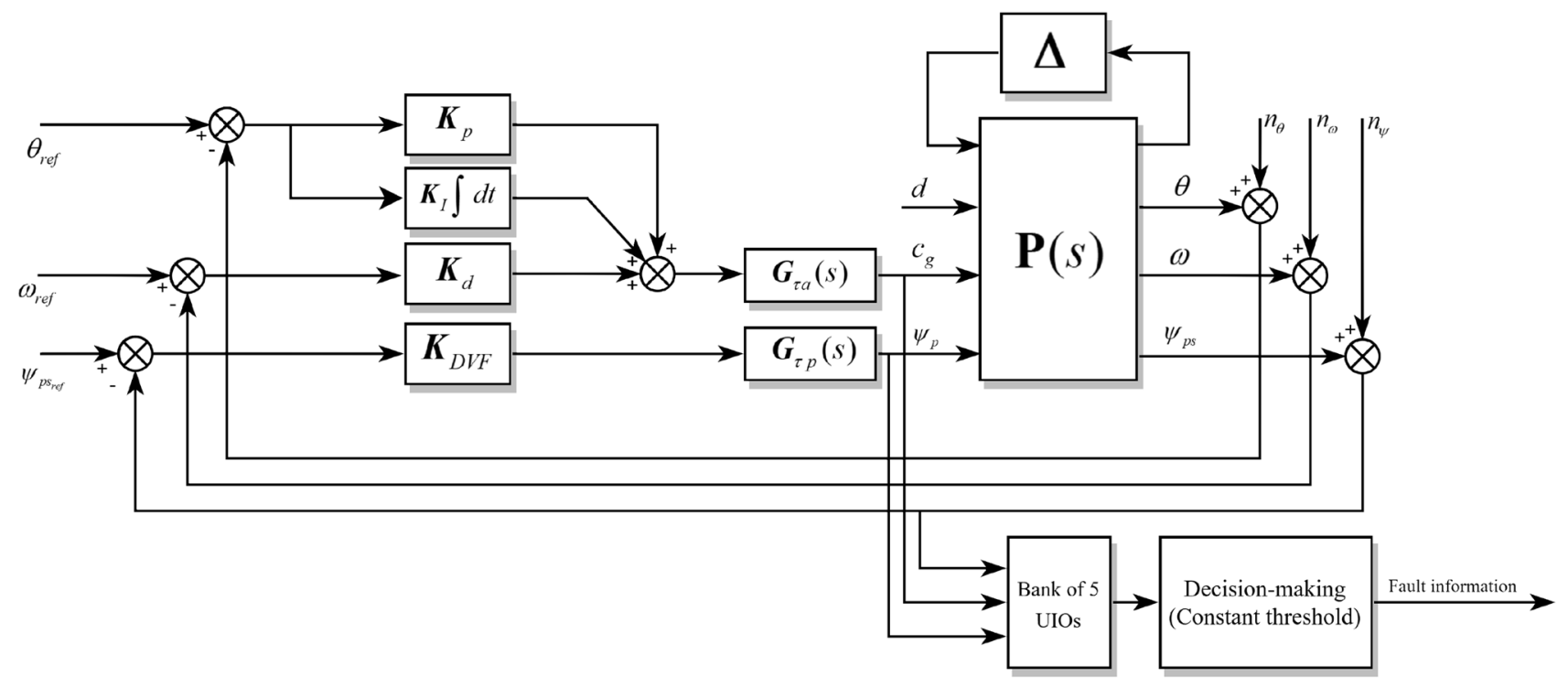

Fig. 7 Closed loop model and failure management unit

Table 5 Plant's uncertainties

\begin{tabular}{ll}
\hline Parameters & Uncertainties \\
\hline Mass & $5 \%$ of N.V \\
{$\left[\mathrm{J}_{x x}, \mathrm{~J}_{y y}, \mathrm{~J}_{z z}\right]$} & Function of mass \\
{$\left[\mathrm{J}_{x y}, \mathrm{~J}_{y z}, \mathrm{~J}_{x z}\right]$} & $5 \%$ of N.V \\
Natural frequencies & $5 \%$ of N.V \\
$c_{P Z}$ & $5 \%$ of N.V
\end{tabular}

N.V. stands for nominal value

Table 6 Measurement noise

\begin{tabular}{ll}
\hline Measures & Noise value \\
\hline Attitude angles & $\mathscr{N}\left(0,1 e-6 \mathrm{deg}^{2}\right)$ \\
Angular velocity & $\mathscr{N}\left(0,4 e-6(\mathrm{deg} / \mathrm{s})^{2}\right)$ \\
Piezo-sensor output voltage & $\mathscr{N}\left(0,5 e-4 V^{2}\right)$ \\
\hline
\end{tabular}

\subsection{Flight Control Law and Fault Modelling}

Let us consider a manoeuvre that excites the most significant elastic modes of the mesh reflector. The gains of the PID for platform attitude control have been selected to reach desired attitude state $\left[\varphi_{r e f}, \theta_{r e f}, \psi_{r e f}\right]=\left[10^{\circ}, 20^{\circ}, 30^{\circ}\right]$ in about $150 \mathrm{~s}$, as shown in Fig. 8. Figure 9 shows a comparison between the angular rates obtained while considering the structure as passive (red curve) with respect to the case in which the distributed network of smart actuators/sensors is activated (blue curve) in the first $5 \mathrm{~s}$ of the manueuver.

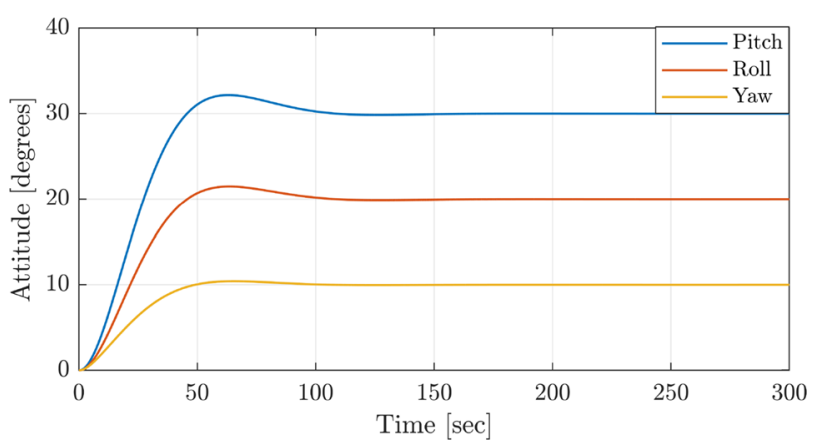

Fig. 8 Attitude of the spacecraft

In this study, the following piezo-actuator fault scenarios have been considered:

- Scenario A: a failure in the piezoelectric device is considered so that the actuator is completely switched off at a certain time instant (in this study, the failure is supposed to occur at $t=1 \mathrm{~s}$ ), thus providing zero control on the structural system.

- Scenario B: a failure in the control system is assumed; therefore, one actuator is fed with a non-zero constant control voltage (instant of the failure $t=2 \mathrm{~s}$ ).

The two scenarios are illustrated in Figs. 10 and 11, where the nominal actuators voltages are reported in black, and the voltage of the failed actuator is represented in red. 

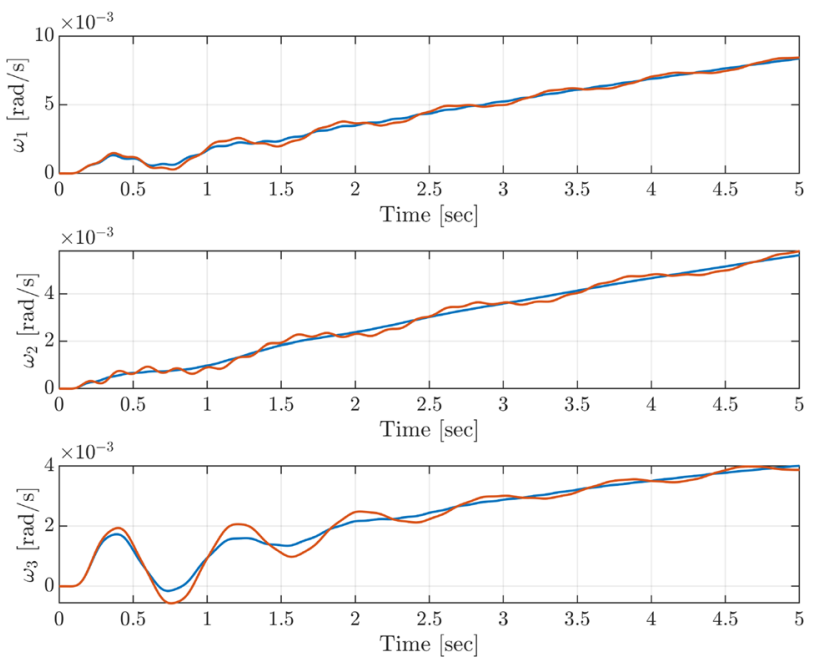

Fig. 9 Angular velocities: passive structure (in red), active structure (in blue)

\subsection{Results: Nominal Model}

First, the performance of the UIOs for both piezo-actuator fault scenarios are tested on the nominal linear plant of the system. Figures 12 and 13 show the behaviour of the L2-norm of the estimation error respectively for Scenario A and Scenario B in case the actuator \#5 fails. Clearly, as described in Sect. 2.2, it possible to define both the threshold value and the confirmation time to promptly detect and isolate the failure. Furthermore, we observe that, as expected, in the nominal case the failure of actuator \#5 can be perfectly decoupled from the output of the fifth observer.

Similar results, not reported here for the sake of brevity, are obtained when using the L2-norm of the derivative of the estimation error.

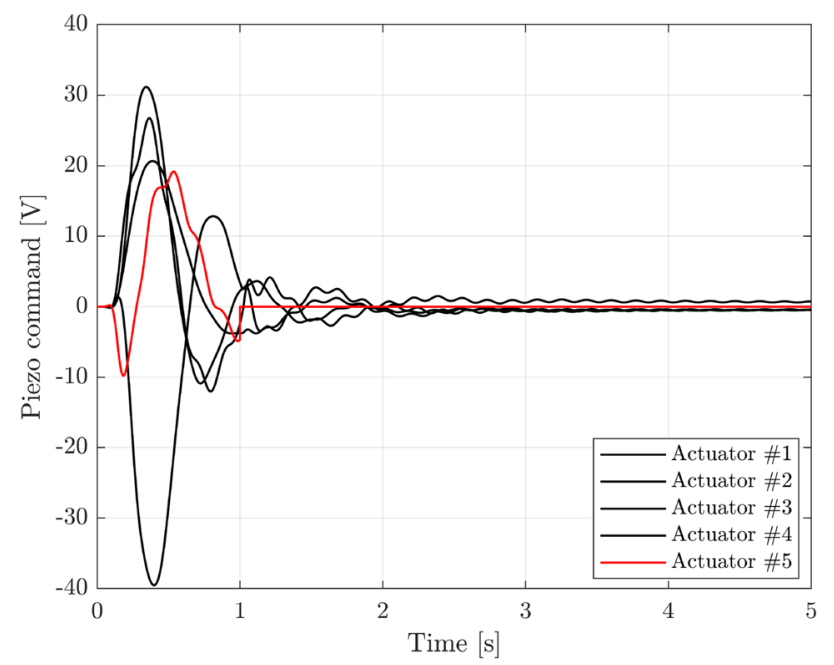

Fig. 10 Failure scenario A

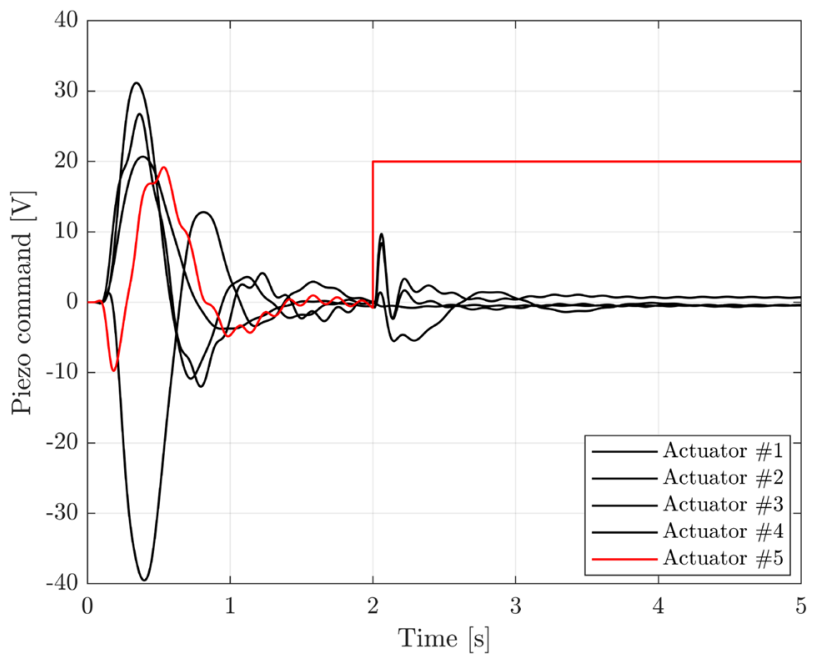

Fig. 11 Failure scenario B

\subsection{Results (Scenario A): Uncertain Model with the Presence of Measurement Noise}

As shown in Fig. 14, as soon as uncertainties on the model are considered, the perfect decoupling of the UIO from its corresponding failure is not valid anymore. Furthermore, since it is meaningful to study the failure of the Scenario A only in the first seconds of the manoeuvre (since reasonable value of voltages are given in the time interval $[0,2]$ seconds as shown in Fig. 10 the detection and isolation is compromised by the transient dynamics of the observer in presence of uncertainties.

Similar conclusion can be drawn, although not reported, if the FDI procedure is done via the derivative of the L2-norm of the estimation error.

\subsection{Results (Scenario B): Uncertain Model with the Presence of Measurement Noise}

In this section, a comparison for the failure of the Scenario B is performed between the two FDI procedures (the L2-norm of the signals $\left\|e_{y i}\right\|, i=1,2, \ldots, 5$ and the one involving the L2-norm of the derivative). In this case, since a failure in Scenario B can happen at any time during the operative life of the system, it has been assumed that a fault on the actuator \#5 occurs at $t=2 \mathrm{~s}$. It is worth noting that also in this configuration it is not possible to detect and isolate a failure if that happens in the first instants of the manoeuvre.

Compared to Scenario A, it is possible to appreciate that, at the instant of the fault, each observer of the bank responds correctly to the failure. However, due to the uncertainties, the measurement noise and the very low magnitude of the response of some observers, as shown in Fig. 15, it is practically impossible to define an optimal 

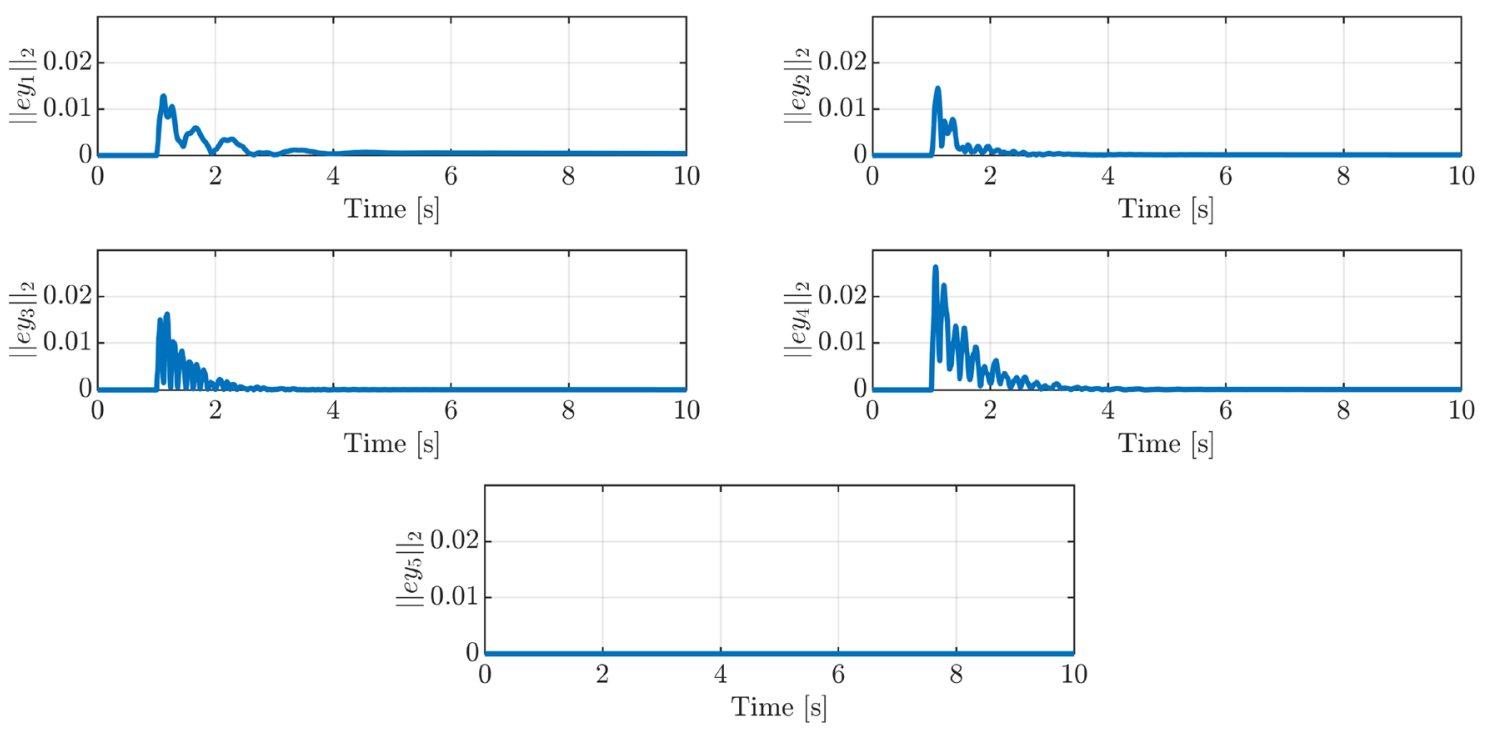

Fig. 12 L2-norm of the estimation error (Scenario A-nominal model)
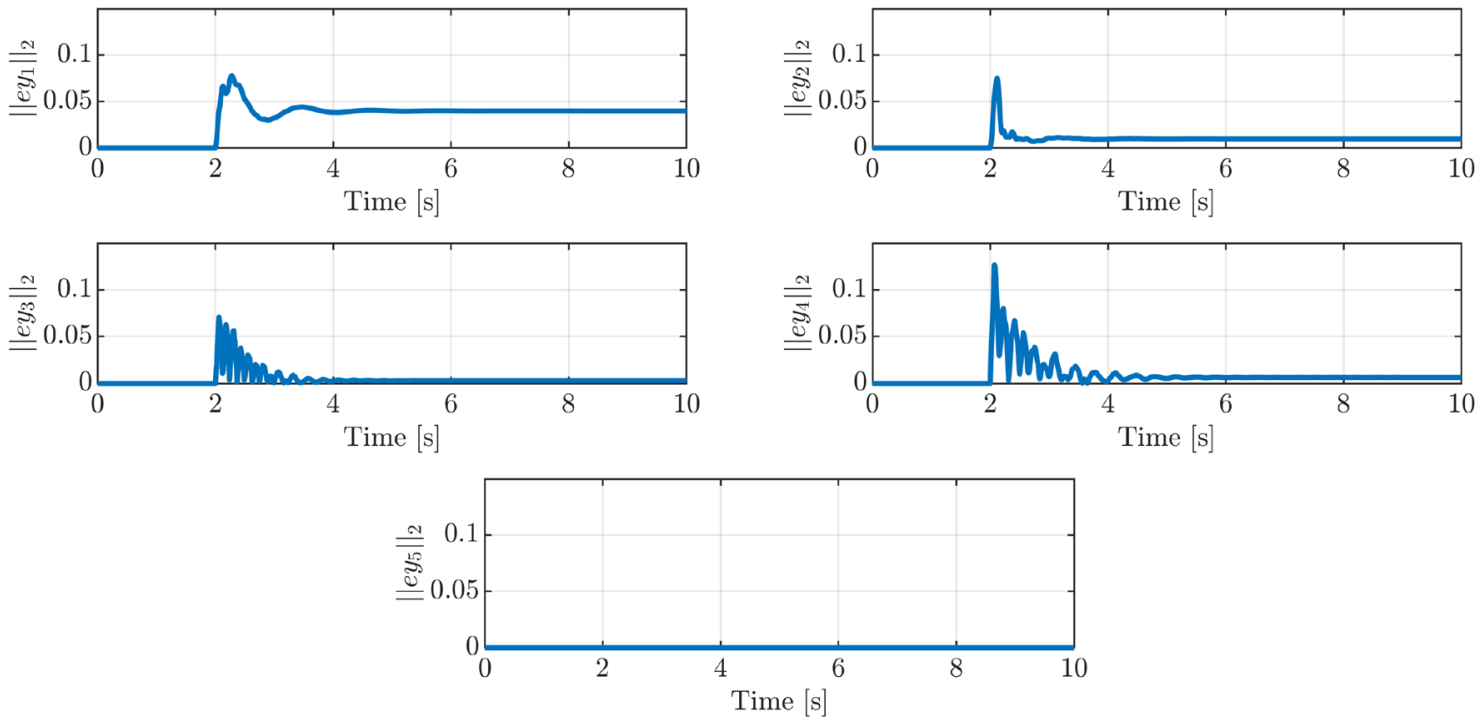

Fig. 13 L2-norm of the estimation error (Scenario B-nominal model)

threshold for the detection and isolation of the failure. On the other hand, by implementing the method involving the derivative of the L2-norm of the estimation error we can see an increase in the amplitude of the output in correspondence of the failure, as shown in Fig. 16, yielding an easier definition of the threshold values.

It should be noted that doing this operation means exchanging an increase in performance in terms of sensitivity to observer failure (higher is better) with the need to lower the confirmation time thus increasing the possibility of false alarms in the process of identifying the action.

Finally, the robustness against modelling uncertainties and measurement noise has been assessed based on an extensive Monte Carlo simulation campaign. The individual failure of each actuator (failure Scenario B) is investigated by randomly sampling the uncertain state space system and the results of the analysis, in terms of percentage of successful failure isolations, successful detections (but no isolation) 

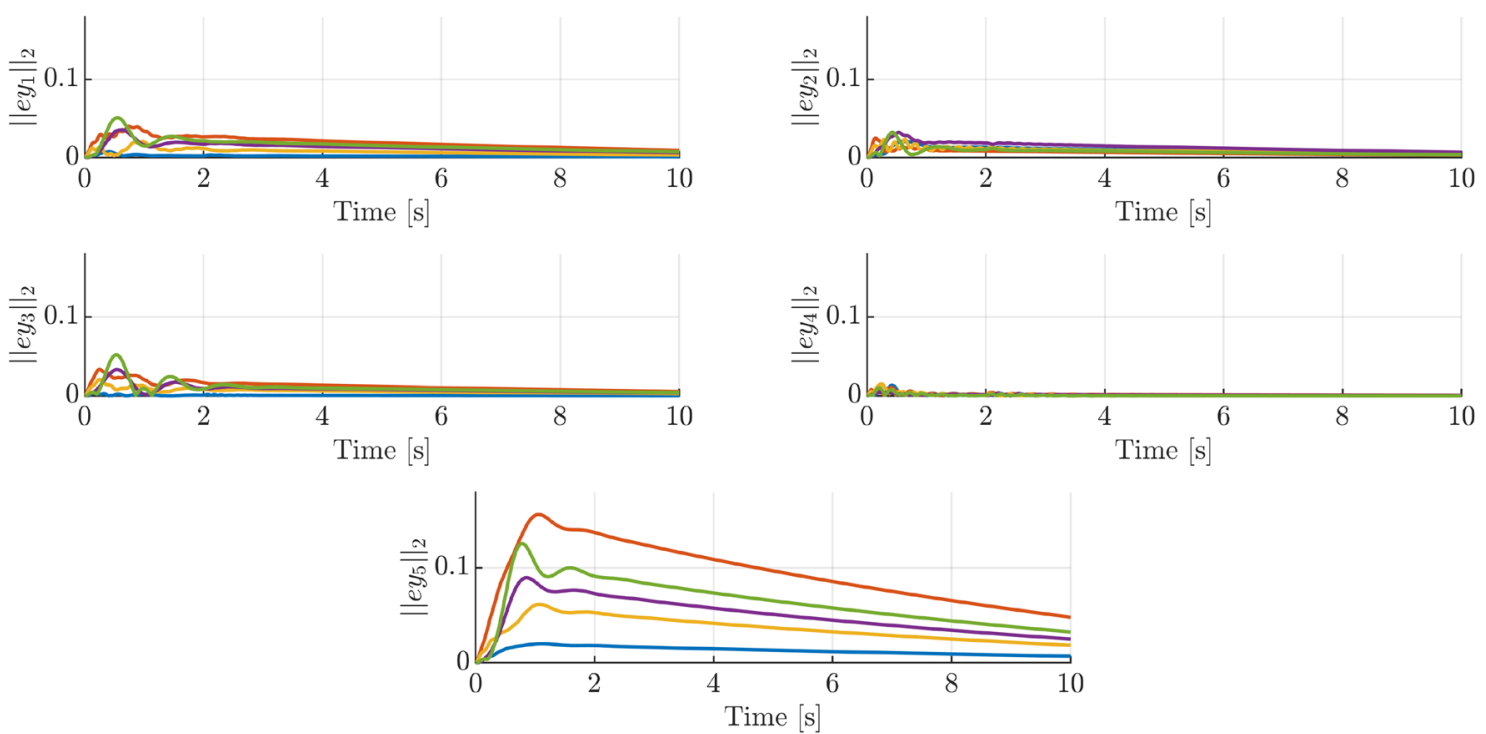

Fig. 14 L2-norm of the estimation error (Scenario A-failure in actuator \#5)
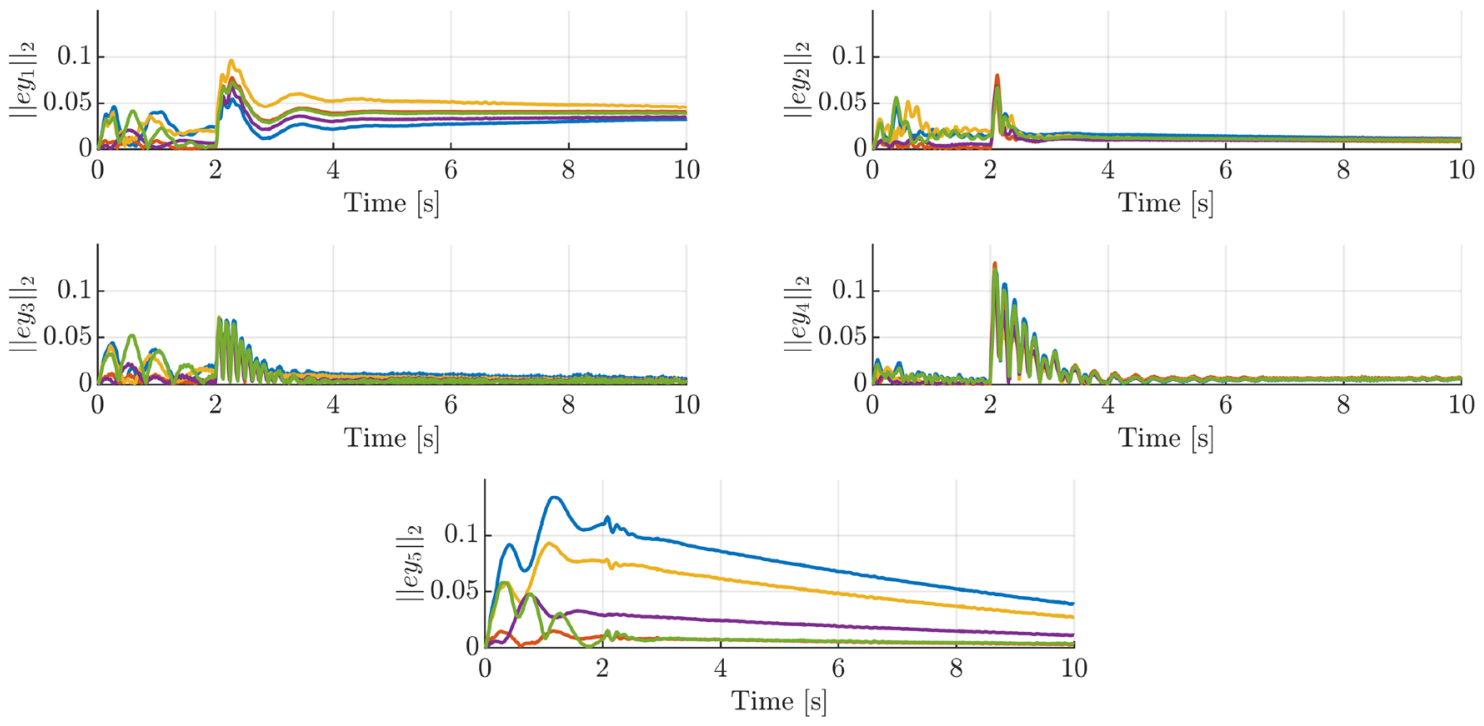

Fig. 15 L2-norm of the estimation error (Scenario B-failure in actuator \#5)

and number of no detection/false alarms, are reported in Table 7 . The campaign is run after an a priori manual tuning of the threshold value and confirmation time, which have been set as $T_{n}=[0.1,0.15,0.1,0.1,0.15]$ and $t_{c}=0.05 \mathrm{~s}$. The analysis shows that in case of a failure in the actuators \#2, \#3 and \#5 the proposed FDI scheme manages almost all the time to isolate or at least detect (meaning that the failure can be circumscribed to a small set of actuators) the failure. In the case of individual failures on actuator \#1 and \#4 the results of the campaign are not optimal.

\section{Conclusions}

This paper presents the implementation of an FDI architecture capable of detecting and isolating the possible failure of a piezoelectric actuator used for active vibration control of a flexible spacecraft in orbit. The interesting aspect of this study lies in the fact that-while the literature contains a large body of work on various fault diagnostic systems for common attitude control subsystem actuators (such as reaction wheels, control moment gyroscopes and 

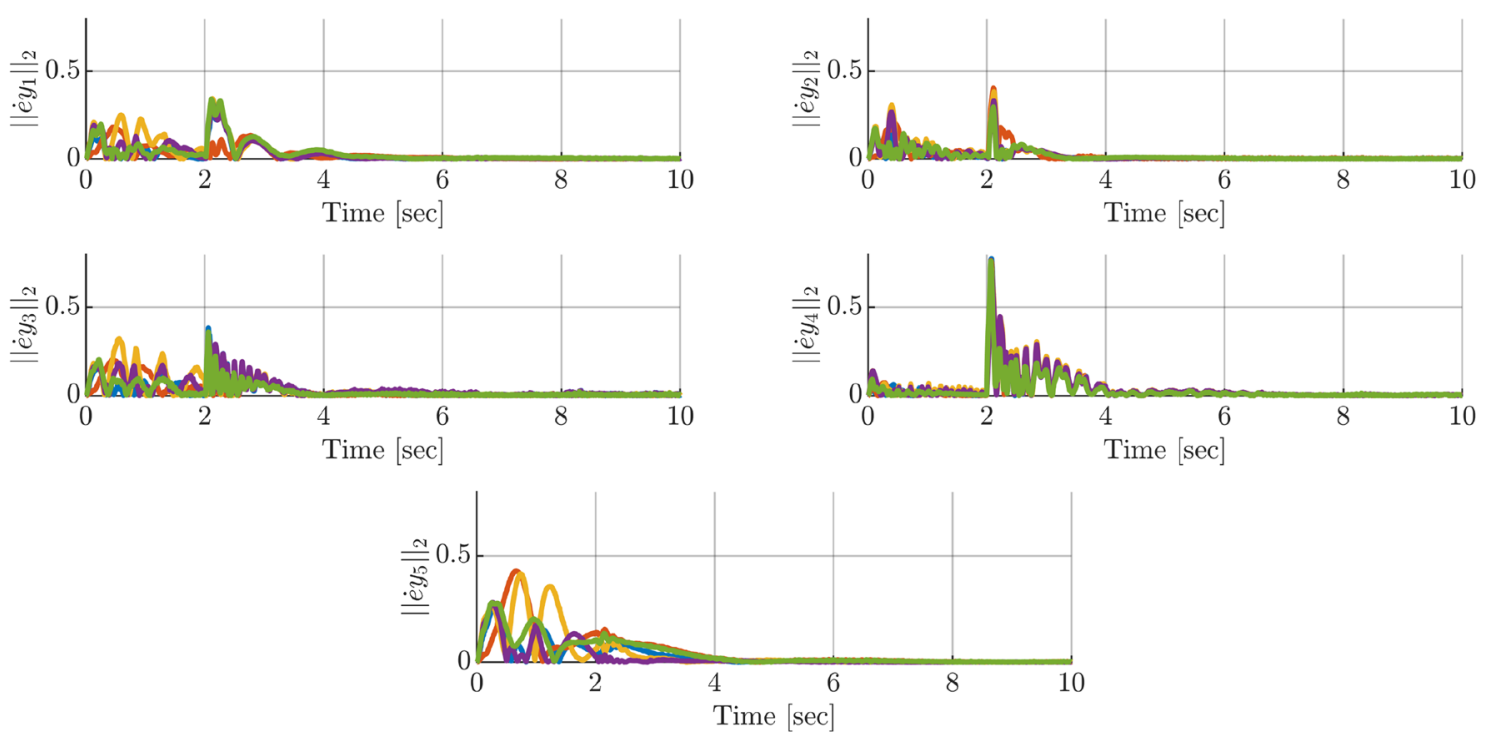

Fig. 16 L2-norm of the estimation error derivative (Scenario B-failure in actuator \#5)

Table 7 Results from the Monte Carlo campaign (200 samples per failure)

\begin{tabular}{llll}
\hline $\begin{array}{l}\text { Actuator } \\
\text { ID (failure) }\end{array}$ & Failure Isolated & Failure only detected $^{\mathrm{a}}$ & $\begin{array}{l}\text { No detec- } \\
\text { tion /false } \\
\text { alarm }\end{array}$ \\
\hline 1 & $43(21.5 \%)$ & $84(42.0 \%)$ & $73(36.5 \%)$ \\
2 & $146(73.0 \%)$ & $54(27.0 \%)$ & $0(0 \%)$ \\
3 & $176(88.0 \%)$ & $23(11.5 \%)$ & $1(0.5 \%)$ \\
4 & $37(18.5 \%)$ & $59(29.5 \%)$ & $104(52.0 \%)$ \\
5 & $200(100 \%)$ & $0(0 \%)$ & $0(0 \%)$ \\
\hline
\end{tabular}

${ }^{a}$ In this column only the cases in which the failures are detected but not isolated are counted

magnetorquers) - the development of fault diagnostic systems for distributed active vibration control in space structures has not been-to the authors' knowledge-yet extensively addressed.

Furthermore, while in attitude control the failure of an actuator (i.e. thruster stuck open) greatly influence the state dynamics and so the output of the observer, in the case of piezo-actuator, although a failure could bring important reduction in the performance of the spacecraft, the effect on the overall dynamics could be rather subtle and could be potentially concealed by other effect such as uncertainties and noise. It is also worth noting that, since each piezoelectric actuator has a contribution on each modal amplitude, the failure of one of them does not directly imply a loss of controllability of the flexible dynamics, whereas the elastic displacements will be still damped, with less efficiency, by the remanent set of actuators.
The simulations of the FDI architecture have shown promising results; however, it is still necessary to verify the proposed strategy in the framework of the non-linear system dynamics. Further developments could also concern the definition of a proper and systematic procedure for the tuning of the identification parameters (thresholds and confirmation time) to both improve the detection and isolation rate and decrease false alarms/no detection percentage. A possible further solution is in the investigation of adaptive thresholds method in which a time-varying threshold [35] is determined for the decision-making process. Moreover, additional studies may be carried out to re-design the FDI unit with increased sensitivity to failure and improved robustness to uncertainties, thus potentially allowing also to tackle the problem of loss of actuator's efficiency.

Funding Open access funding provided by Università degli Studi di Roma La Sapienza within the CRUI-CARE Agreement.

Open Access This article is licensed under a Creative Commons Attribution 4.0 International License, which permits use, sharing, adaptation, distribution and reproduction in any medium or format, as long as you give appropriate credit to the original author(s) and the source, provide a link to the Creative Commons licence, and indicate if changes were made. The images or other third party material in this article are included in the article's Creative Commons licence, unless indicated otherwise in a credit line to the material. If material is not included in the article's Creative Commons licence and your intended use is not permitted by statutory regulation or exceeds the permitted use, you will need to obtain permission directly from the copyright holder. To view a copy of this licence, visit http://creativecommons.org/licenses/by/4.0/. 


\section{references}

1. Osder, S.: Practical view of redundancy management application and theory. J. Guid. Control Dyn. (1999). https://doi.org/10. 2514/2.4363

2. Ding, S.X.: Model-based fault diagnosis techniques: design schemes, algorithms and tools, 2nd edn. Springer-Verlag, London (2013)

3. Patton, R.J.: Robust model-based fault diagnosis: the state of the ART. IFAC Proc. Vol. (1994). https://doi.org/10.1016/S14746670(17)47997-3

4. Henry, D., LePeuvédic, C., Strippoli, L., Ankersen, F.: Robust model-based fault diagnosis of thruster faults in spacecraft. Safeprocess (2015). https://doi.org/10.1016/j.ifacol.2015.09.670

5. Fonod, R., Henry, D., Charbonnel, C., Bornschlegl, E.: Position and attitude model-based thruster fault diagnosis: a comparison study. J. Guid. Control Dyn. (2015). https://doi.org/10.2514/1. G000309

6. Tudoroiu, N., Sobhani-Tehrani, E., Khorasani, K.: Interactive bank of unscented Kalman filters for fault detection and isolation in reaction wheel actuators of satellite attitude control system. In: IECON 2006-32nd Annual Conference on IEEE Industrial Electronics (2006). https://doi.org/10.1109/IECON.2006.347890

7. Chen, W., Saif, M.: Observer-based fault diagnosis of satellite systems subject to time-varying thruster faults. ASME. J. Dyn. Syst. Meas. Control. (2007). https://doi.org/10.1115/1.2719773

8. Patton, R., Uppal, F., Simani, S., Polle, B.: Robust FDI applied to thruster faults of a satellite system. IFAC Proc. Vol. (2007). https://doi.org/10.3182/20070625-5-FR-2916.00002

9. Valdes, A., Khorasani, K.: A pulsed plasma thruster fault detection and isolation strategy for formation flying of satellites. Appl. Soft Comput. (2010). https://doi.org/10.1016/j.asoc.2009.09.005

10. Fu, K., Zhao, Z., Ren, G., Xiao, Y., Feng, T., Yang, J., Gasbarri, P.: From multiscale modeling to design of synchronization mechanisms in mesh antennas. Acta Astronaut. (2019). https://doi.org/ 10.1016/j.actaastro.2019.03.056

11. Patton, R.J., Chen, J., Nielsen, S.B.: Model-based methods for fault diagnosis: some guide-lines. Trans. Inst. Meas. Control (1995). https://doi.org/10.1177/014233129501700203

12. Willsky, A., Jones, H.: A generalized likelihood ratio approach to the detection and estimation of jumps in linear systems. IEEE Trans. Autom. Control (1976). https://doi.org/10.1109/TAC.1976. 1101146

13. Basseville, M., Nikiforov, I.V.: Detection of abrupt changes: theory and application. Prentice Hall (1993). https://doi.org/10.1080/ 00401706.1994.10485821

14. Chen, W., Saif, M.: Unknown input observer design for a class of nonlinear systems: an LMI approach. 2006 American Control Conference (2006). https://doi.org/10.1109/ACC.2006.1655461

15. Fonod, R., Henry, D., Charbonnel, C., Bornschlegl, E., Losa, D., Bennani, S.: Robust FDI for fault-tolerant thrust allocation with application to spacecraft rendezvous. Control. Eng. Pract. (2015). https://doi.org/10.1016/j.conengprac.2015.05.004

16. Chilali, M., Gahinet, P.: Ho design with pole placement constraints: an LMI approach. IEEE Trans. Autom. Control (1996). https://doi.org/10.1109/9.486637

17. Henry, D., Ankersen, F., Strippoli, L.: Chapter 9-a class of unknown input observers under $\mathrm{H}^{\infty}$ performance for fault diagnosis: application to the mars sample return mission. Emerging methodologies and applications in modelling, new trends in observer-based control. Academic Press (2019). https://doi.org/ 10.1016/B978-0-12-817034-2.00022-8

18. Astro Aerospace: AstroMesh ${ }^{\mathrm{TM}}$ deployable reflector data sheet DS-409 07/04. Northrop Grumman Space Technology (2004)
19. Morterolle, S., Maurin, B., Dube, J.F., Averseng, J., Quirant, J.: Modal behavior of a new large reflector conceptual design. Aerosp. Sci. Technol. (2015). https://doi.org/10.1016/j.ast.2015. 01.002

20. Angeletti, F., Iannelli, P., Gasbarri, P., Sabatini, M.: End-to-end design of a robust attitude control and vibration suppression system for large space smart structures. Acta Astronaut. (2021). https://doi.org/10.1016/j.actaastro.2021.04.007

21. Angeletti, F., Gasbarri, P., Sabatini, M., Iannelli, P.: Design and performance assessment of a distributed vibration suppression system of a large flexible antenna during attitude manoeuvres. Acta Astronaut. (2020). https://doi.org/10.1016/j.actaastro.2020. 04.015

22. Gasbarri, P., Monti, R., De Angelis, C., Sabatini, M.: Effects of uncertainties and flexible dynamic contributions on the control of a spacecraft full-coupled model. Acta Astronaut. (2014). https:// doi.org/10.1016/j.actaastro.2012.08.018

23. Piéfort, V., Loix, N., Preumont, A.: Modeling of piezolaminated composite shells for vibration control. Proc. Eur. Conf. Spacecraft Struct. Mater. Mech. Test. 428, 525-530 (1999)

24. Xu, R., Li, D., Jiang, J., Zou, J.: Decentralized adaptive fuzzy vibration control of smart gossamer space structure. J. Intell. Mater. Syst. Struct. (2017). https://doi.org/10.1177/1045389X16 679023

25. Wang, W., Yang, Z.: A compact piezoelectric stack actuator and its simulation in vibration control. Tsinghua Sci. Technol. (2009). https://doi.org/10.1016/S1007-0214(10)70029-8

26. Mazzini, L.: The dynamics of the flexible satellite. In: flexible spacecraft dynamics, Control and Guidance. Aerospace Technology Springer (2016)

27. Ankersen, F.: Guidance, navigation, control and relative dynamics for spacecraft proximity Maneuvers. Doctoral thesis. Aalbord University. (2015). https://vbn.aau.dk/ws/portalfiles/portal/61599 334/finnAnkersenThesis.pdf. Accessed 12 May 2021

28. Alazar, D., Cumer C., Tantawi, K. H. M.: Linear dynamic modeling of spacecraft with various flexible appendages and on-board angular momentums. In: 7th International ESA conference on guidance, navigation and control systems (GNC 2008). 2 June 2008-5 June 2008 (Tralee, Ireland). https://oatao.univ-toulouse. fr/1899/. Accessed 12 May 2021

29. Texas Instruments, Application Report. Signal conditioning piezoelectric sensors. SLOA033A. (2020). https://www.ti.com/lit/an/ sloa033a/sloa033a.pdf. Accessed 12 May 2021

30. Balas, M.J.: Direct velocity feedback control of large space structures. J. Guid. Control (1979). https://doi.org/10.2514/3.55869

31. Hanta, V., Prochazka, A.: Rational approximation of time delay. https://www2.humusoft.cz/www/papers/tcp09/035_hanta.pdf. Accessed 12 May 2021

32. Zhou, K., Doyle, J.C., Glover, K.: Robust and optimal control. Prentice Hall, Upper Saddle river (1996)

33. Löfberg, J.: YALMIP: a toolbox for modeling and optimization in MATLAB. IEEE Int. Conf. Robot. Autom. Conf. (2004). https:// doi.org/10.1109/CACSD.2004.1393890

34. Toh, K.C., Todd, M.J., Tütüncü, R.H.: SDPT3-a matlab software package for semidefinite programming, version 1.3. Optimiz. Methods Softw. (1999). https://doi.org/10.1080/1055678990 8805762

35. Emami-Naeini, A., Akhter, M.M., Rock, S.M.: Effect of model uncertainty of failure detection: the threshold selector. IEEE Trans. Autom. Control (1988). https://doi.org/10.1109/9.14432

Publisher's Note Springer Nature remains neutral with regard to jurisdictional claims in published maps and institutional affiliations. 